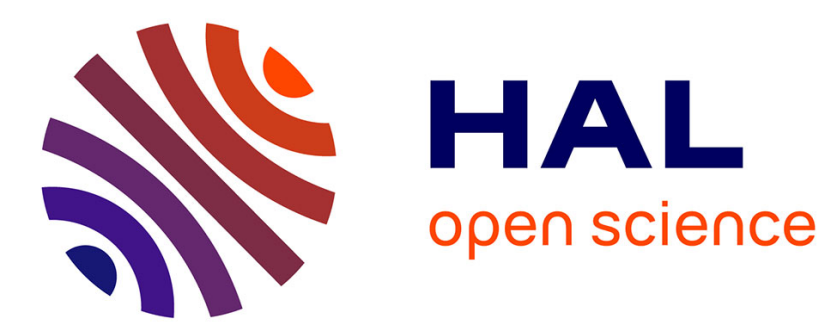

\title{
Etude de systèmes d'interaction piétons-voitures autonomes
}

\author{
Emeline Ah-Tchine, Erica De Vries
}

\section{To cite this version:}

Emeline Ah-Tchine, Erica De Vries. Etude de systèmes d'interaction piétons-voitures autonomes. Actes de la 32e conférence francophone sur l'Interaction Humain-Machine (IHM'20.21), Apr 2021, Virtual Event, France. pp.7:1-6, 10.1145/3450522.3451253 . hal-03567059

\section{HAL Id: hal-03567059 \\ https://hal.science/hal-03567059}

Submitted on 11 Feb 2022

HAL is a multi-disciplinary open access archive for the deposit and dissemination of scientific research documents, whether they are published or not. The documents may come from teaching and research institutions in France or abroad, or from public or private research centers.
L'archive ouverte pluridisciplinaire HAL, est destinée au dépôt et à la diffusion de documents scientifiques de niveau recherche, publiés ou non, émanant des établissements d'enseignement et de recherche français ou étrangers, des laboratoires publics ou privés. 


\title{
Etude de systèmes d'interaction piétons-voitures autonomes
}

\author{
Study of Interaction Systems between Pedestrians and Autonomous Cars
}

\author{
Emeline Ah-tchine \\ Univ. Grenoble Alpes, Laboratoire de Recherche sur les \\ Apprentissages en Contexte(LaRAC), 1251 Avenue \\ Centrale, 38400 Saint-Martin-d'Hères - \\ ahtchine.e@gmail.com
}

\begin{abstract}
For any innovative artefact, the modalities of future use should be considered. Communication between road users and autonomous cars is one of the primary conditions to ensure safety. The focus is on pedestrian, the most vulnerable users. Two interactive systems were studied. The first system informs the pedestrian about the speed of the car, the second system gives advice using pedestrian traffic lights. A study on the comprehension of these two systems was conducted with open-ended questions about out-of-context pictures and multiple choice questions about contextual videos. Comprehension was assessed using three criteria: the speed of the vehicle, pedestrian traffic lights and right of way, and the road crossing decision regarding pedestrian rule compliance. Results show that the advice system allows less interpretation and instigates road crossing decisions in compliance with road safety rules. The informative system is only relevant for change of speed questions.
\end{abstract}

\section{CCS CONCEPTS}

- Human-centered computing; • Human computer interaction; • HCI design and evaluation methods;

\section{KEYWORDS}

communication, evaluation, comprehension

\section{RÉSUMÉ}

Pour tout objet issu de l'innovation, des modalités d'usage doivent être envisagés. La communication entre les usagers de la route et une voiture autonome est une des conditions primordiales pour assurer la sécurité de tous. Nous nous focalisons sur les usagers les plus vulnérables que sont les piétons. Deux systèmes d'interaction ont été étudiés. Le premier système a pour but d'informer le piéton en affichant la vitesse du véhicule, le deuxième système conseille le piéton en affichant des feux piétons. Une étude de la compréhension de ces systèmes d'interactions a été réalisée avec des questions ouvertes à partir d'images sans contexte ainsi qu'avec des questions à choix multiples à partir de vidéos contextuelles. La compréhension a été évaluée selon trois critères : le changement de la vitesse du

IHM '20.21, April 13-16, 2021, Virtual Event, France

(C) 2021 Copyright held by the owner/author(s). Publication rights licensed to ACM. This is the author's version of the work. It is posted here for your personal use. Not for redistribution. The definitive Version of Record was published in Actes de la 32e conférence Francophone sur l'interaction Humain-Machine (IHM'20.21), April 13-16, 2021, Virtual Event, France. https://doi.org/10.1145/3450522.3451253

\author{
Erica de Vries \\ Univ. Grenoble Alpes, Laboratoire de Recherche sur les \\ Apprentissages en Contexte(LaRAC), 1251 Avenue \\ Centrale, 38400 Saint-Martin-d'Hères - \\ Erica.deVries@univ-grenoble-alpes.fr
}

véhicule, les feux piétons et la priorité au piéton, et la décision de traverser en accord avec le code de la route. Les résultats montrent que le système qui conseille laisse moins de place à l'interprétation et permet davantage de comportements de traversée conformes au code de la route. Le système qui informe n'est pertinent que sur des questions de vitesse.

\section{MOTS-CLÉS}

conception, design participatif, créativité, communication, évaluation, compréhension

\section{INTRODUCTION}

Pour tout nouvel objet issu de l'innovation, un mode d'interaction avec les individus est nécessaire pour pouvoir faire l'usage de l'objet [1]. Cependant, les interactions pensées en amont lors de la conception ne sont pas forcément intuitives pour les utilisateurs se trouvant devant le produit fini. Souvent certains modes d'interactions se révèlent obsolètes dans l'usage. Dans le domaine de la signalisation routière, on peut citer l'exemple de l'échec d'un dispositif de signalisation. Il s'agit de la mise en place de demi-sphères de béton blanc sur fond blanc pour décourager l'utilisation d'une piste cyclable par les automobilistes. Ces demi-sphères se sont avérées être dangereuses plutôt que sécurisantes pour les cyclistes. Pour contrer ces problèmes, les pratiques du design peuvent inclure les usagers cibles dans le processus de création d'artefact.

Le contexte de l'environnement routier est particulier, il demande aux usagers une prudence accrue avec des décisions qui doivent être précises et rapides. L'arrivée de la voiture autonome entend apporter de la sécurité notamment en ce qui concerne les usagers les plus vulnérables que sont les piétons. Cependant même si les piétons sont prioritaires, il s'agit de pouvoir établir un bon partage de la route. Ainsi, il s'agit de déterminer la capacité de systèmes d'interactions d'une voiture totalement autonome de permettre de prendre des décisions et d'émettre des comportements conformes au code de la route.

L'évaluation d'un artefact concerne la plupart du temps trois dimensions : l'utilité, l'utilisabilité et l'acceptabilité [2-4]. L'utilité se réfère à la capacité de l'artefact à permettre à l'utilisateur d'atteindre les objectifs établis. L'utilisabilité rend compte de la 
facilité d'utilisation du produit en termes d'apprentissage ou de documentation. L'acceptabilité rend compte de l'intention de l'usage de l'artefact. Dans le cas de l'interaction piétons-voitures autonomes, on parle de réceptivité car le piéton ne fait pas un usage du véhicule mais interagit avec lui [5].

La présente contribution comporte la conception de systèmes d'interaction de la voiture autonome dans une séance d'élaboration par des experts, ainsi qu'une étude en termes de compréhension par des utilisateurs futurs.

\subsection{Les systèmes d'interaction pour la voiture autonome}

La communication entre piétons et chauffeur de véhicule est variée et complexe. Stanciu et al. [6] font une revue des modes de communication entre piétons et véhicules en distinguant les signaux formels et informels. L'utilisation des clignotants pour indiquer un changement de direction, le bras tendu d'un cycliste sont des signaux formels. Les signaux informels sont ceux appris par l'observation et l'expérience, comme le contact visuel, et dépendent beaucoup du contexte et de la culture. Dans une étude proactive, Rothenbücher et al. [7] ont utilisé une voiture dont le conducteur était rendu invisible en étant déguisé en siège. Même si le comportement de traversée des piétons ne semblait pas être influencé, des moments d'hésitation et un sentiment d'incertitude quant au comportement du véhicule ont été relevés. Cette observation amène à des questionnements sur la forme et le contenu de potentiels systèmes d'interaction de la voiture autonome $[7,8]$.

Les constructeurs de voiture autonome ainsi que les chercheurs ont été proactifs sur ce sujet. De nombreux clips promotionnels sur des prototypes de systèmes d'interaction peuvent être consultés par le grand public. Les systèmes d'interaction proposés par les constructeurs se veulent novateurs : équiper la voiture d'yeux [9], de sourires, ou encore d'émoticônes. Au niveau de la recherche, différents fonctionnements de systèmes d'interaction ont été étudiés. Par exemple, Li et al. [10] ont étudié l'efficacité d'un code couleur pour la perception de l'urgence. C'est également le cas de Rad et al. [11] qui ont testé un système d'éclairage de la voiture autonome : jaune pour un piéton pris en compte, vert pour une voiture qui laisse passer et rouge pour une voiture qui ne s'arrête pas (danger). Même si ce système a été démontré comme informatif pour les piétons, la signification des différentes couleurs a prêté à confusion. Par exemple, la couleur rouge peut être interprétée comme un arrêt du véhicule ou un danger pour le piéton. D’autres chercheurs ont fait l'étude de systèmes plus aboutis. Mahadevan et al. [12] font la comparaison de solutions visuelles, auditives et physiques. En ce qui concerne la forme, les résultats n'ont pas démontré une préférence particulière pour une modalité sensorielle. Au niveau du contenu, les informations jugées comme nécessaires sont la prise en compte du piéton et le comportement du véhicule. Clamann et al. [13] ont comparés des messages disposés sur le parechoc d'un van considéré comme autonome. Les messages indiquaient soit la vitesse du véhicule soit une indication sur la sécurité d'une traversée. Les résultats montrent qu'il n'y a pas d'influence des messages sur le temps de prise de décision de traverser. Cependant, la présence d'un expérimentateur dans le véhicule a pu biaiser les résultats. L'équipe de De Clerc et al. [14] a comparé deux messages (le piéton peut passer/le piéton ne peut pas passer) sous différentes formes : des points lumineux variant horizontalement (knightrider), du texte, une barre horizontale se transformant en sourire et une condition contrôle. Dans cette étude, la présence de systèmes d'interaction participe au sentiment de sécurité encourageant la traversée de rue. Cependant, certains types de messages nécessitent une phase d'apprentissage afin d'être compris.

\subsection{Etude de la compréhension des systèmes d'interaction}

L'objectif de systèmes d'interaction de la voiture autonome est de permettre aux piétons d'interagir avec une voiture entièrement contrôlée par une machine. On considère que cette interaction est efficace si le piéton comprend et perçoit le contenu des messages émis par la voiture autonome. Cependant, des messages conçus par un groupe de personnes peuvent être sujets à des interprétations individuelles différentes [15]. De plus, on peut se poser la question de la validité des solutions de communications pour un objectif donné. En effet, une même représentation peut amener à différents raisonnements individuels $[15,16]$, il est donc nécessaire de s'assurer que les solutions amènent aux comportements attendus, autrement dit que les usagers comprennent la signification et savent comment agir [17]. Il s'agit d'évaluer l'utilisabilité de ces systèmes par la qualité de la compréhension des utilisateurs. Une bonne compréhension des systèmes d'interaction se traduit par des décisions et les comportements en accord avec ce que préconise le code de la route.

L'évaluation de la compréhension peut s'organiser selon plusieurs méthodes. Wolff et Wogalter [18] ont comparé une évaluation de la compréhension de symboles routiers en faisant varier le contexte (avec et sans représentation d'un environnement contextuel) et la méthode d'évaluation (question ouverte et questions à choix multiples). La présence de contexte facilite la compréhension, cependant ce résultat est modulé par le nombre de réponses possibles dans les questions à choix multiples.

Dans notre étude, nous comparons la compréhension des systèmes d'interactions de façon isolée sous forme d'images avec des questions ouvertes et de manière contextualisé sous forme de vidéos avec des questions à choix multiples. Les questions ouvertes avec des images non contextualisées vont permettre de recueillir des descriptions libres et isolées. De cette façon, nous pourrons apprécier la nature et la quantité de propositions explicatives alternatives. Les vidéos contextualisées avec un choix imposé de réponses pourront récolter des réponses spontanées telle qu'attendue dans un contexte routier pressant. Il s'agit de pouvoir remplir les trois fonctions d'un système de sécurité: décrire une situation, prescrire et/ou interdire une action [17].

Les systèmes d'interaction étudiés représentent la vitesse du véhicule par des valeurs numériques. La priorité du piéton est représentée par des icônes de feux piétons avec un code couleur associé. Cette dualité correspond à des représentations figuratives et descriptives. Les représentations descriptives utilisent des symboles qui n'ont pas de lien avec la forme physique d'un objet, comme par exemple la représentation de la vitesse de la voiture par des chiffres. Les représentations figuratives ont des similarités avec les objets de référence, cette définition correspond au système d'interaction 
utilisant les feux piétons. De plus, les représentations descriptives sont adéquates pour la prédiction d'évènements, ou encore pour orienter l'attention d'un individu [19]. Les représentations figuratives quant à elles facilitent davantage la compréhension de scénarios ou d'évènements. En effet, selon le modèle intégratif de compréhension de texte et d'image de Schnotz et Bannert [20], une représentation figurative est plus facilement assimilable à une représentation interne. De plus, les représentations ayant une similarité physique avec l'objet de référence améliorent la compréhension [21]. Nous faisons donc l'hypothèse que le système d'interaction qui conseille dispose de plus d'éléments permettant une meilleure compréhension que le système qui informe.

\section{MÉTHODE}

\subsection{Elaboration de deux systèmes d'interaction de la voiture autonome par des experts}

Une séance d'élaboration a été organisée dans les locaux d'Ideas laboratory. En tant que living lab [22], Ideas laboratory dispose d'une pièce modulable pour le travail de groupe dans un objectif de création. Un groupe d'experts s'est réuni afin de convenir de deux systèmes d'interaction. Pour ce faire, des critères ont été établis pour une utilisabilité maximale dans un contexte de traversée de rue par des piétons. Premièrement, il s'agissait de convenir d'un système visible par un piéton positionné sur le trottoir voyant arriver la voiture de loin. La position du système devait correspondre à l'endroit où le contact visuel avec un chauffeur humain est généralement établi. Deuxièmement, il s'agissait de considérer un système d'interaction dynamique qui s'adapte aux changements de la situation routière. Un processus dynamique est préconisé dans des environnements où les informations sont changeantes [23]. Troisièmement, en vue de l'implémentation rapide sur un prototype, les experts devaient s'appuyer sur un système réalisable avec des technologies existantes. Enfin, le dispositif devait permettre de signaler le caractère autonome de la voiture. Huit experts ont été conviés pour une réunion de deux heures. Le groupe comprenait des conseillers scientifiques, des responsables innovations ouvertes, un directeur développement et mobilité, un chargé de projets de marketing de l'innovation, ainsi qu'un développeur en réalité virtuelle.

Les solutions proposées rejoignent les différentes propositions commerciales des constructeurs de la voiture autonome. Les deux systèmes d'interaction retenus sont des messages sur un écran d'information situé sur le toit de la voiture autonome. Le message affiche soit une information sur la vitesse de la voiture avec une indication sur son accélération ou son freinage, soit un conseil sur la conduite du piéton à tenir vis-à-vis de la voiture (priorité ou refus de priorité). Ces deux systèmes d'interaction se retrouvent étudiés dans la littérature comme étant deux types d'informations pertinentes établies par des focus groupes [24].

Le groupe des experts s'est appuyé sur les critères définis en amont pour décider du contenu et de la forme finale des systèmes d'interaction de la voiture autonome. Premièrement, concernant le type de solution technique, un écran de forme rectangulaire a été choisi pour diffuser des messages visibles de loin. Deuxièmement, deux types de contenus ont été retenus et décrits par les experts : information «la vitesse du véhicule a un but informatif, l'interface

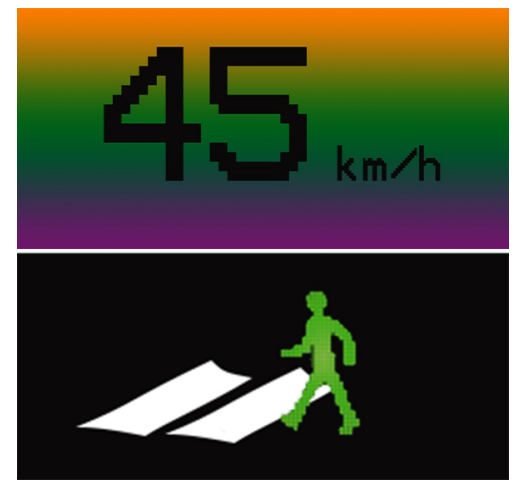

Figure 1: Prototypes des deux systèmes d'interaction de la voiture autonome

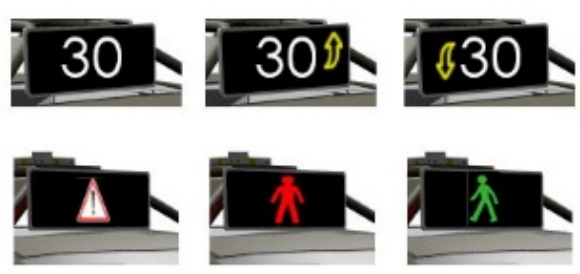

Figure 2: : Deux systèmes d'interaction Information (vitesse) et Conseil (feux piétons)

permettra d'indiquer une accélération ou une diminution de la vitesse. »et conseil «l'indication sur la priorité du piéton fera office de conseil, le code couleur des feux piétons sera utilisé : vert pour indiquer une priorité au piéton et rouge pour refus de priorité. ». Et enfin, concernant la forme, ces messages seront semblables aux panneaux à messages variables disponibles dans l'infrastructure routière, i.e. sous forme de symboles et d'affichage numérique. La figure 1 montre une illustration élaborée par l'animatrice de la séance.

Un développeur en informatique s'est chargé de numériser les deux systèmes d'interaction retenus pour des véhicules autonomes en réalité virtuelle (Figure 2). Le premier système informe le piéton en indiquant la vitesse du véhicule en temps réel. Une absence de flèche indique une vitesse constante. Une flèche jaune montante indique une accélération, une flèche jaune descendante indique un freinage. Le deuxième système conseille le piéton à l'aide d'icônes similaires aux feux piétons : Un panneau de signalisation avec un point d'exclamation indique un danger (faire attention), un feu vert indique la priorité au piéton (traverser), un feu rouge indique un refus de priorité (ne pas traverser).

\subsection{Echantillon}

201 participants ont été recrutés contre rémunération financière au sein de l'UFR de Sciences Humaines et Sociales d'une université française. L'échantillon avait une moyenne d'âge de 22.08 ans, avec un écart-type de 5.70 ans, pour une étendue de 19-64 ans avec $80 \%$ de femmes. $84.6 \%$ ont indiqué avoir obtenu le code de la route, $72.1 \%$ ont obtenu le permis B dont $55.2 \%$ en permis probatoire. 
Table 1: Réponses correctes selon le code de la route pour les deux systèmes d'interaction et la condition contrôle pour les questions ouvertes $(\mathrm{QO})$ et les questions à choix multiples

\begin{tabular}{|c|c|c|c|}
\hline Comportement & Changement de vitesse & $\begin{array}{l}\text { Catégorie } \\
\text { Feux piétons/priorité }\end{array}$ & Décision \\
\hline \multirow{4}{*}{$\begin{array}{l}\text { Vitesse constante } \\
13 \quad 1\end{array}$} & Vitesse constante (QO) & Danger (QO) & \multirow{4}{*}{ Je ne traverse pas } \\
\hline & On ne peut pas le déterminer & Elle ne me laisse pas passer & \\
\hline & Autre & On ne peut pas le déterminer & \\
\hline & & Autre & \\
\hline \multirow{2}{*}{$\begin{array}{l}\text { Accélération } \\
\text { 13仓 }\end{array}$} & La vitesse augmente (QO) & Feu rouge $(\mathrm{QO})$ & \multirow[t]{2}{*}{ Je ne traverse pas } \\
\hline & Elle accélère & Elle ne me laisse pas passer & \\
\hline \multirow{2}{*}{$\begin{array}{l}\text { Décélération } \\
\text { अ13 } \AA\end{array}$} & La vitesse diminue (QO) & Feu vert $(\mathrm{QO})$ & \multirow[t]{2}{*}{ Je traverse } \\
\hline & Elle ralentit & Elle me laisse passer & \\
\hline
\end{tabular}

\subsection{Contextualisation et modalité de réponse}

La compréhension a été mesurée à partir d'images (hors contexte) et à partir de vidéos (de façon contextualisée). Pour la compréhension à partir d'images, trois questions ouvertes ont été posées pour chacune des six images de la Figure $1: 1$ ) Qu'est-ce que cela représente ? 2) Qu'est-ce que la voiture veut vous communiquer par cet affichage ? et 3) Qu'est-ce que cela implique pour vous en tant que piéton?

Neuf vidéos ont été réalisées en réalité virtuelle : six vidéos pour chacune des images de la Figure 2 et trois vidéos pour la condition contrôle avec une voiture sans système d'interaction à allure constante, en accélération, et en décélération. Chaque séquence vidéo est prise du point de vue d'un piéton sur le trottoir voyant arriver une voiture autonome. La vidéo se termine avant que la voiture n'arrive à la hauteur du piéton. Pour la compréhension à partir de vidéos, trois questions à choix multiples ont été posées pour chacune des neuf vidéos. Les questions concernaient le changement de vitesse, la priorité et la décision de traverser : 1) Dans cette vidéo, la voiture autonome voudrait m'indiquer que... (Elle ralentit, Elle accélère, On ne peut pas le déterminer, Autre (précisez)). 2) Dans cette vidéo, la voiture autonome voudrait m'indiquer que... (Elle me laisse passer, Elle ne me laisse pas passer, On ne peut pas le déterminer, Autre (précisez)). 3) Par rapport à la situation que vous venez de visionner, quelle action entreprendriez-vous en réponse? (Je traverse, Je ne traverse pas, Autre (précisez)).

\subsection{Mesure de la compréhension}

Pour les questions ouvertes comme pour les questions à choix multiples, les réponses ont été jaugées dans trois catégories par rapport 1) au changement de la vitesse de la voiture, 2) aux feux piétons et/ou la priorité et 3) à la décision de traverser (tableau 1). Pour les questions ouvertes, une grille d'analyse a permis de relever les unités correspondant dans chaque catégorie [25]. Pour les questions à choix multiples, il s'agit des alternatives de réponses. Un point est obtenu pour chaque bonne réponse. Ainsi, le score de compréhension va de 0 à 3 dans chaque catégorie (addition des trois comportements).

\subsection{Procédure}

Les participants étaient accueillis dans une salle et dirigés vers un poste informatique désigné par un numéro d'anonymat, et signaient un formulaire de consentement. La première partie consistait à un test de lecture de chiffres (pour la détection du daltonisme). La deuxième partie présentait le contexte de l'étude. Les participants répondaient alors aux deux questionnaires de compréhension. Pour les questions ouvertes à partir d'images, les deux systèmes d'interaction étaient contrebalancés. Pour les questions à choix multiples à partir des vidéos, les neufs vidéo ont été passés dans deux ordres prédéterminés. Enfin, les participants répondaient à quelques questions démographiques et une page de débriefing clôturait la séance. Les participants obtenaient un bon pour leur rémunération.

\section{RÉSULTATS}

\subsection{Compréhension hors contexte à partir d'images}

A l'aide de la grille d'analyse, nous avons d'abord relevés des réponses alternatives originales. Celles-ci sont variées pour le système Information (vitesse). Le message sur la vitesse du véhicule $\mathrm{a}$ été confondu avec l'indication d'une vitesse maximale, une durée ou une consommation de carburant. Par ailleurs, les flèches montantes et descendantes ont été parfois perçues comme un changement de direction de la voiture. Les messages du système Conseil (feux piétons) n'ont pas suscités d'interprétations déviantes.

Le Tableau 2 résume les scores de compréhension pour les deux modalités d'évaluation de la compréhension des systèmes d'interaction. Les bonnes réponses ont été établies en fonction de ce que préconise le code de la route, les scores de compréhension vont de 0 à 3 points, 3 points étant le score maximum de bonnes réponses (voir Tableau 1). Les questions ouvertes n'ont pas incité de réponses dans chaque catégorie (moyennes proches de zéro). Ainsi, des feux piétons ou la priorité ne sont pas mentionnés lorsque la voiture affiche des chiffres, et inversement, des changements de vitesse ne sont pas mentionnés lorsque la voiture affiche des feux piétons. Le système Conseil (feux piétons) résulte en un score très élevé de compréhension. En effet, le pourcentage de bonnes 
Table 2: Moyennes (écart type) des scores de compréhension

\begin{tabular}{llccr}
\hline Contexte & Système d'interaction & Changement de vitesse & Feux piétons/priorité & Décision de traverser \\
\hline A partir d'images & Information (vitesse) & $2.10(1.05)$ & $0.01(0.14)$ & $1.69(0.99)$ \\
& Conseil (feux piétons) & $0.00(0.00)$ & $2.89(0.32)$ & $2.95(0.24)$ \\
A partir de vidéos & Contrôle & $1.49(0.62)$ & $1.66(0.67)$ & $2.08(0.45)$ \\
& Information (vitesse) & $2.88(0.36)$ & $2.22(0.62)$ & $2.24(0.49)$ \\
& Conseil (feux piétons) & $1.74(0.62)$ & $2.86(0.37)$ & $2.68(0.49)$ \\
\hline
\end{tabular}

réponses est supérieur à $94 \%$ pour les trois comportements (triangle de danger, le feu rouge, et le feu vert). Le système Information (vitesse) a été compris un peu moins bien ( $90 \%$ pour la vitesse constante, $60 \%$ pour l'accélération et $60 \%$ pour la décélération). Enfin, les réponses concernant les décisions de traverser sont davantage en accord avec les règles du code de la route pour le système Conseil que pour le système Information $(t(200)=-18.051, p<.000)$.

\subsection{Compréhension en contexte à partir de vidéos}

Une analyse de variance a été réalisée pour les trois catégories de réponse avec le système d'interaction en intra sujets. L'effet du système d'interaction était significatif pour toutes les catégories (changement de vitesse de la voiture $(F(2,199)=422.634, p<.005)$, feux piétons/priorité $(F(2,199)=274.628, p<.005)$, décisions de traverser $(F(2,199)=98.489, p<.005))$. Par ailleurs, les paramètres testés suivent le contraste d'Helmert (contrôle versus les deux systèmes d'interaction et les deux systèmes entre eux). Les contrastes étaient significatifs. Le Tableau 2 montre que les deux systèmes obtiennent de meilleurs scores de compréhension que l'absence de système. Le système Information (vitesse) permet le mieux d'apprécier le changement de vitesse, alors que le système Conseil permet de mieux comprendre la priorité du piéton. Les deux systèmes d'interaction renseignent mieux qu'une absence de message, et chaque système d'interaction est approprié dans sa catégorie de messages visés (information ou conseil).

\section{CONCLUSION}

L'objectif de cette étude était d'évaluer la compréhension de système d'interactions de la voiture autonome. Bien que ces systèmes furent élaborés avec l'intention de communiquer tout un ensemble d'information sur le comportement de la voiture, les résultats montrent que les messages des systèmes renseignent bien sur le comportement de la voiture qu'ils illustrent. Cependant, le système d'interaction qui informe avec la vitesse du véhicule obtient plus d'interprétations alternatives que le système qui conseille. Les feux piétons étant présentement des messages à l'attention des piétons, un effet de familiarité peut expliquer ce plus grand score de compréhension et le nombre plus faible d'interprétation de sa signification [26]. De plus, le système qui conseille est le plus pertinent pour des décisions de traverser en accord avec le code de la route. Un système d'interaction possédant des éléments familiers du code de la route et qui permet de construire un raisonnement de manière rapide pour une prise de décision immédiate semble le plus approprié pour l'environnement routier.
Il a quelques limites à prendre en compte pour cette étude. Il est très difficile de pouvoir séparer la forme et le contenu pour des dispositifs d'interface homme-machine. Ainsi, dans cette étude, l'analyse de ces deux dimensions a été réalisée simultanément sans croiser les variables et les modes d'évaluation.

Une comparaison entre la compréhension de systèmes d'interaction et les comportements de décision associée pourrait davantage compléter nos résultats.

L'évaluation de la compréhension d'interactions hommemachine est nécessaire pour évaluer leur adéquation au regard de la situation et des actions prescrites. Cependant, l'évaluation de la compréhension en tant que vérification de l'utilisabilité des systèmes d'interactions est une première étape dans le processus. Les concepts d'utilité et d'acceptabilité complètent l'évaluation de solutions. Une prochaine étude pourrait considérer une évaluation complète des systèmes d'interactions.

Pour parfaire notre démarche d'évaluation, il s'agirait de pouvoir observer les comportements d'usage de ces systèmes d'interaction en utilisant les méthodes de la réalité virtuelle dans un environnement réaliste et immersif [27].

L'enjeu des interactions homme-machine et plus particulièrement les interactions piétons-voiture autonome est de pouvoir trouver une solution de communication qui peut se substituer aux interactions entre humains tout en étant la plus intuitive possible. En effet, certaines interactions informelles entre chauffeur et piétons sont maintenant ancrées, comme devenues une extension d'un répertoire d'information du comportement des voitures. Cependant, la difficulté réside dans le développement d'interfaces polyvalentes qui permettent le maintien d'un partage de la route entre différents usagers et la voiture autonome pour toutes situations routières.

\section{RÉFÉRENCES}

[1] Mallein, P., \& Tarozzi, S. (2002). Des signaux d'usage pertinents pour la conception des objets communicants. Les Cahiers du numérique, Vol. 3(4), 61-70.

[2] Grislin, M., \& Kolski, C. (1996). Evaluation des interfaces homme-machine lors du développement de système interactif. Technique et Science informatiques, 15(3), 265-296.

[3] Senach, B. (1990). Evaluation ergonomique des interfaces homme-machine: Une revue de la littérature.

[4] Tricot, A., Plégat-Soutjis, F., Camps, J.-F., Amiel, A., Lutz, G., \& Morcillo, A. (2003). Utilité, utilisabilité, acceptabilité : Interpréter les relations entre trois dimensions de l'évaluation des EIAH. In: Environnements Informatiques pour l'Apprentissage Humain. 391-402.

[5] Deb, S., Strawderman, L., Carruth, D. W., DuBien, J., Smith, B., \& Garrison, T. M. (2017). Development and validation of a questionnaire to assess pedestrian receptivity toward fully autonomous vehicles. Transportation research part $C$ : emerging technologies, 84, 178-195.

[6] Stanciu, S. C., Eby, D. W., Molnar, L. J., St. Louis, R. M., Zanier, N., \& Kostyniuk, L. P. (2018). Pedestrians/Bicyclists and Autonomous Vehicles: How Will They Communicate? Transportation Research Record, 0361198118777091. https://doi. org $/ 10.1177 / 0361198118777091$ 
[7] Rothenbücher, D., Li, J., Sirkin, D., Mok, B., \& Ju, W. (2015). Ghost driver: A platform for investigating interactions between pedestrians and driverless vehicles. Adjunct Proceedings of the 7th International Conference on Automotive User Interfaces and Interactive Vehicular Applications, 44-49. http://dl.acm.org/citation. cfm?id=2809755

[8] Gupta, S., Vasardani, M., \& Winter, S. (2016). Conventionalized Gestures for the Interaction of People in Traffic with Autonomous Vehicles. Proceedings of the 9th ACM SIGSPATIAL International Workshop on Computational Transportation Science, 55-60. https://doi.org/10.1145/3003965.3003967

[9] Chang, C.-M., Toda, K., Sakamoto, D., \& Igarashi, T. (2016). Eyes on a Car: An interface design for communication between an autonomous car and a pedestrian. 9th ACM International conference on Automotive User Interfaces and Interactive Vehicular Applications, Oldenburg, Germany. https://dl.acm.org/citation.cfm?id= 3122989

[10] Li, Y., Dikmen, M., Hussein, T. G., Wang, Y., \& Burns, C. (2018). To Cross or Not to Cross: Urgency-Based External Warning Displays on Autonomous Vehicles to Improve Pedestrian Crossing Safety. Proceedings of the 10th International Conference on Automotive User Interfaces and Interactive Vehicular Applications, 188-197. https://doi.org/10.1145/3239060.3239082

[11] Rad, S. R., de Almeida Correia, G. H., \& Hagenzieker, M. (2020). Pedestrians' road crossing behaviour in front of automated vehicles: Results from a pedestrian simulation experiment using agent-based modelling. Transportation research part F: traffic psychology and behaviour, 69, 101-119.

[12] Mahadevan, K., Somanath, S., \& Sharlin, E. (2018, avril 21). Communicating Awareness and Intent in Autonomous Vehicle-Pedestrian Interaction. CHI 2018, Montréal, QC, Canada. https://dl.acm.org/citation.cfm?doid=3173574.3174003

[13] Clamann, M., Aubert, M., \& Cummings, M. L. (2017). Evaluation of Vehicle-toPedestrian Communication Displays for Autonomous Vehicles. Transportation Research Board 96th Annual Meeting Transportation Research Board. https: //trid.trb.org/View/1437891

[14] de Clercq, K., Dietrich, A., Núñez Velasco, J. P., de Winter, J., \& Happee, R. (2019). External Human-Machine Interfaces on Automated Vehicles: Effects on Pedestrian Crossing Decisions. Human Factors, 61(8), 1351-1370. https://doi.org/ 10.1177/0018720819836343

[15] De Vries, E., \& Masclet, C. (2013). A framework for the study of external representations in collaborative design settings. International fournal of Human-Computer
Studies, 71(1), 46-58.

[16] De Vries, E. (2011). Learning with external representations. In N. M. Seel (Éd.), Encyclopedia of the Sciences of Learning (p. 2016-2019). Springer Science \& Business Media.

[17] Tijus, C., Barcenilla, J., De Lavalette, B. C., \& Meunier, J.-G. (2007). The design, understanding and usage of pictograms. Studies in writing, 21, 17.

[18] Wolff, J. S., \& Wogalter, M. S. (1998). Comprehension of pictorial symbols: Effects of context and test method. Human factors, 40(2), 173-186.

[19] Schnotz, W., Baadte, C., \& Müller, A. (2010). Creative thinking and problem solving with depictive and descriptive representations. In Use of representations in reasoning and problem solving (p. 21-45). Routledge.

[20] Schnotz, W., \& Bannert, M. (2003). Construction and interference in learning from multiple representation. Learning and instruction, 13(2), 141-156.

[21] Shinar, D., Dewar, R. E., Summala, H., \& Zakowska, L. (2003). Traffic sign symbol comprehension: A cross-cultural study. Ergonomics, 46(15), 1549-1565. https: //doi.org/10.1080/0014013032000121615

[22] Dell'Era, C., \& Landoni, P. (2014). Living Lab : A methodology between usercentred design and participatory design. Creativity and Innovation Management, 23(2), 137-154.

[23] Groff, J., Boucheix, J.-M., Lowe, R. K., Argon, S., Saby, L., Alauzet, A., \& PaireFicout, L. (2014). Don't miss your train! Just follow the computer screen animation : Comprehension processes of animated public information graphics. Computers in Human Behavior, 30, 206-221.

[24] Ackermann, C., Beggiato, M., Schubert, S., \& Krems, J. F. (2019). An experimental study to investigate design and assessment criteria : What is important for communication between pedestrians and automated vehicles? Applied Ergonomics, 75, 272-282. https://doi.org/10.1016/j.apergo.2018.11.002

[25] Chi, M. T. (1997). Quantifying qualitative analyses of verbal data: A practical guide. The journal of the learning sciences, 6(3), 271-315.

[26] Ben-Bassat, T., \& Shinar, D. (2006). Ergonomic Guidelines for Traffic Sign Design Increase Sign Comprehension. Human Factors, 48(1), 182-195. https://doi.org/10. 1518/001872006776412298

[27] Ah-tchine. E., De Vries, E. (2020). Information or advice? A virtual reality study of message content for road crossing in front of autonomous cars. Le travail humain, 83(4), 407-427. 